\title{
EXPERIMENTAL STUDIES OF THE NONLINEAR MOMENTUM COMPACTION FACTOR AT BESSY II *
}

\author{
J. Feikes, G. Wüstefeld ${ }^{\dagger}$, BESSY GmbH \\ Rudower Chaussee 5, 12489 Berlin, Germany
}

\begin{abstract}
Experimental studies were performed, to measure the nonlinear behavior of the momentum compaction factor $\alpha$ at the BESSY II electron storage ring. Based on these results the momentum acceptance of the storage ring can be derived. Several sets of measurements at different values of the horizontal chromaticity in the range of $\Delta Q_{x} /\left(\Delta p / p_{0}\right)=\xi_{x}=-5.5 \ldots+8.1$ were generated. Depending on tune and horizontal chromaticity particles in the range from $-4 \%<\Delta p / p_{0}<8 \%$ could be stored.
\end{abstract}

\section{INTRODUCTION}

BESSY II is a $1.7 \mathrm{GeV}$, low emittance, high brilliance synchrotron radiation light source recently started operation at Berlin-Adlershof [1]. The lattice of the storage ring is a 16-cell 'Double Bend Achromat' with alternating high and low $\beta$-values and zero dispersion in the straights. The ring circumference is $240 \mathrm{~m}$. The transverse tunes are $Q_{x}=17.8$ and $Q_{y}=6.7$, the natural chromaticities are $\xi_{\text {nat. }, x}=-52.7$ and $\xi_{\text {nat. }, y}=-26.8$.

A source of major life time limitations of the stored electron beam are scattering processes, were electrons suffer a sudden energy change, well known as Touschek effect and bremsstrahlung process [2]. To avoid a loss of these scattered particles and to achieve a good beam lifetime, the transverse and longitudinal momentum acceptance of the machine is designed for $\Delta p / p_{0} \pm 3 \%$. One task of the presented measurements was the determination of the actual machine momentum acceptance.

\section{THE MOMENTUM COMPACTION FACTOR $\alpha$}

\subsection{Definition of $\alpha$}

The length of the particle orbit together with the magnetic field on this orbit defines the energy of the electrons. The rf-frequency and its harmonic number define the path length of the electrons in the ring. Because of the fast radiation damping, the particle energy will follow a change of the rf-frequency or the magnetic field within few milliseconds. In the experiments discussed here the rf-frequency at fixed magnetic field was detuned to change the electron energy.

\footnotetext{
* Work supported by the Bundesministerium für Bildung, Wissenschaft, Forschung und Technologie and by the Land Berlin.

† Email: wuestefeld@bessy.de, feikes@bii.bessy.de
}

To describe changes of the path length for electrons with small momentum deviations it is common, to apply a series expansion of the path length with respect to the momentum deviation [2]. The influence of betatron oscillations on the momentum compaction factor can be ignored here, because theire amplitudes are less than $1 \mathrm{~mm}$. It seems that there is not a unique definition of the type of series expansion in literature. For the following discussion two types of definitions for the momentum compaction factor $\alpha$ are required:

$$
\alpha_{p}=\frac{d L}{d p} / \frac{L}{p} \text {, and } \alpha=\frac{\Delta L}{\Delta p} / \frac{L_{0}}{p_{0}} \text {. }
$$

In case of the first definition $\alpha_{p}=\alpha_{p}(p)$ is based on small changes of the momentum around some specified momentum value $p$. It is given by the differential change of the path length $L$ with respect to the momentum. This $\alpha_{p}$ depends on the local properties of the particle orbit, independent of the reference orbit length $L_{0}$ and reference momentum $p_{0}$. In our experiments $p$ could typically differ from the reference value $p_{0}$ by a few percent, whereas the 'differential' changes are about a factor ten smaller.

The second, similar definition is based on difference expressions, valid for larger momentum changes of the order of few percent with respect to the reference momentum $p_{0}$. It is normalized with respect to the reference values of momentum $p_{0}$ and orbit length $L_{0}$. Both definitions, $\alpha_{p}$ and $\alpha$, are functions of the momentum $p$.

The definitions of $\alpha$ and $\alpha_{p}$ are not independent of each other, they are connected by a differential equation :

$$
\alpha_{p}=\frac{1+\Delta p / p_{0}}{1+\alpha \Delta p / p_{0}} \frac{d\left(\alpha \Delta p / p_{0}\right)}{d\left(\Delta p / p_{0}\right)} .
$$

In the case that $p$ approaches $p_{0}$ both definitions yield the same limiting value $\alpha_{0}$ :

$$
\lim _{p \rightarrow p_{0}} \alpha=\lim _{p \rightarrow p_{0}} \alpha_{p}=\alpha_{0}
$$

Based on linear approximations, this value can be calculated:

$$
\alpha_{0}=\frac{1}{L_{0}} \oint_{\text {ring }} \frac{D_{x}}{\rho} d s,
$$

were $D_{x}$ is the linear dispersion function and $\rho$ the dipole bending radius. For the present storage ring optics we have $\alpha_{0}=7.3 \cdot 10^{-4}$. 


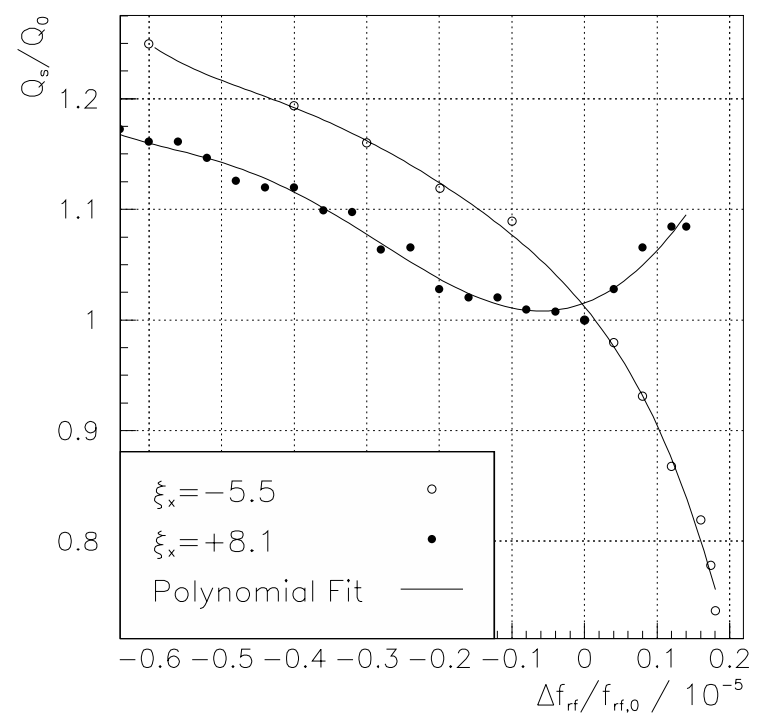

Figure 1: Measured normalized synchrotron tune $Q_{s}$ as a function of the relative rf-frequency change $\left(\Delta f_{r f}\right) / f_{r f, 0}$ for two different values of the horizontal chromaticity $\xi_{x}$. The measured points are fitted by a polynomial (line).

\subsection{Reconstruction of $\alpha$ from measurements}

From the longitudinal beam dynamics the expression for the longitudinal tune $Q_{s}$ of the synchrotron oscillation is given by [2]:

$$
Q_{s, p}^{2}=\frac{h \eta_{p} e V_{c a v} \cos \psi_{p}}{2 \pi \beta_{p} c p}
$$

were $h$ is the harmonic number, $V_{\text {cav }}$ the effective peak Voltage of the rf-frequency, $\psi$ is the phase angle of the synchronous particle and $\beta c$ the velocity of the particle. The index $p$ is used to point out that these values are related to the local properties of the orbit, defined for a given momentum $p$. Only the local orbit dynamics is involved, because the relative energy spread of the bunches is 'differentially' small, less than $10^{-3}$.

The accurate estimate of the effective rf-Voltage seen by the beam is rather difficult. By relating the longitudinal tune to the tune at $p=p_{0}$ the expression can be simplified and becomes independent of the Voltage:

$$
\frac{Q_{s, p}^{2}}{Q_{s, 0}^{2}}=\frac{\eta_{p} \cos \psi_{p}}{\beta_{p} p} / \frac{\eta_{0} \cos \psi_{0}}{\beta_{0} p_{0}}
$$

The phase angle $\psi$ defined by the large amplitude of the rf-Voltage and a comparably small energy loss per turn is only some few degrees. The change of this angle with respect to the few percent changes of the momentum considered here is even smaller and will be neglected, we set $\cos \psi_{0}=\cos \psi_{p}$. At typical BESSY II energies a small change of the particle momentum hardly changes the particle velocity. Therefore, the 'frequency slip factor' $\eta_{p}$ can be well approximated by $-\alpha_{p}$ and $\beta_{p}=\beta_{0}$. Taking into account these approximations yields the simple result:

$$
\frac{Q_{s, p}^{2}}{Q_{s, 0}^{2}}=\frac{\alpha_{p}}{\alpha_{0}} \frac{p_{0}}{p}
$$

The left-hand-side expression involving the synchrotron tunes was measured by varying the rf-frequency, two typical examples of the measurements plotted as $Q_{s, p} / Q_{s, 0}$ are shown in Fig.1. It was shown by simulations, that fluctuations of the measured points could be explained by misalignment errors.

Replacing $\alpha_{p}$ in the equation by its definition and rearranging:

$$
\alpha_{0} \frac{d p}{p_{0}}=\frac{d L}{L} \frac{Q_{s, 0}^{2}}{Q_{s, p}^{2}} .
$$

Integrating both sides yields:

$$
\alpha_{0} \frac{p-p_{0}}{p_{0}}=\int \frac{Q_{s, 0}^{2}}{Q_{s, p}^{2}} \frac{d L}{L} .
$$

The orbit changes can be expressed by appropriate rffrequency changes $\frac{d L}{L}=-\frac{d f_{r f}}{f_{r f}}$, which transforms the integrant into only rf-depended terms:

$$
\alpha_{0} \frac{\Delta p}{p_{0}}=-\int \frac{Q_{s, 0}^{2}}{Q_{s, p}^{2}} \frac{d f_{r f}}{f_{r f}}=-\int \frac{Q_{s, 0}^{2}}{Q_{s, p}^{2}} \frac{d r}{(1+r)} .
$$

For the last step $f_{r f}$ was substituted by $(1+r) f_{r f, 0}$. The whole integrant can be constructed from the measured data and fitted by a polynomial of the type $a_{i}\left(\Delta f_{r f} / f_{r f, 0}\right)^{i}$, similar as shown in Fig.1, were a fit to the original data was applied.

The polynomial can be integrated analytically

$-\alpha_{0} \frac{\Delta p}{p_{0}}=\frac{\Delta f_{r f}}{f_{r f, 0}}+\frac{1}{2} a_{2}\left(\frac{\Delta f_{r f}}{f_{r f, 0}}\right)^{2}+\frac{1}{3} a_{3}\left(\frac{\Delta f_{r f}}{f_{r f, 0}}\right)^{3}+\ldots$

and the relative momentum deviation as a function of the relative rf-detuning is obtained.

The integration smoothes the measured data, at this point results of measured, fitted and simulated data are compared, see Fig.2. Results are shown for two examples with the most detuned chromaticities, at $\xi_{x}=+8.1$ and $\xi_{x}=-5.5$. Similar results from data for $\xi_{x}=+5.6,+2.2,-1.6$ and -5.2 are not presented here. The simulation was done with the beam optical code MAD [3] applying some minor adjustments, such as a $1 \%$ change of $\alpha_{0}$ and a small shift of the absolute momentum scale.

The polynomial fit has the advantage, that critical numerical expressions of small numerator and denominator, such as $\left(\Delta f_{r f} / f_{r f, 0}\right) /\left(\Delta p / p_{0}\right)$ can be calculated analytically. A second fit applied to the reverse function $r=r\left(\Delta p / p_{0}\right)$ yields the rf-detuning and the change of the orbit length as a function of the momentum deviation:

$-\frac{\Delta f_{r f}}{f_{r f, 0}}=\frac{\Delta L}{L_{0}}=\frac{\Delta p}{p_{0}}\left(\alpha_{0}+\alpha_{1}\left(\frac{\Delta p}{p_{0}}\right)+\alpha_{2}\left(\frac{\Delta p}{p_{0}}\right)^{2}+\ldots\right)$. 


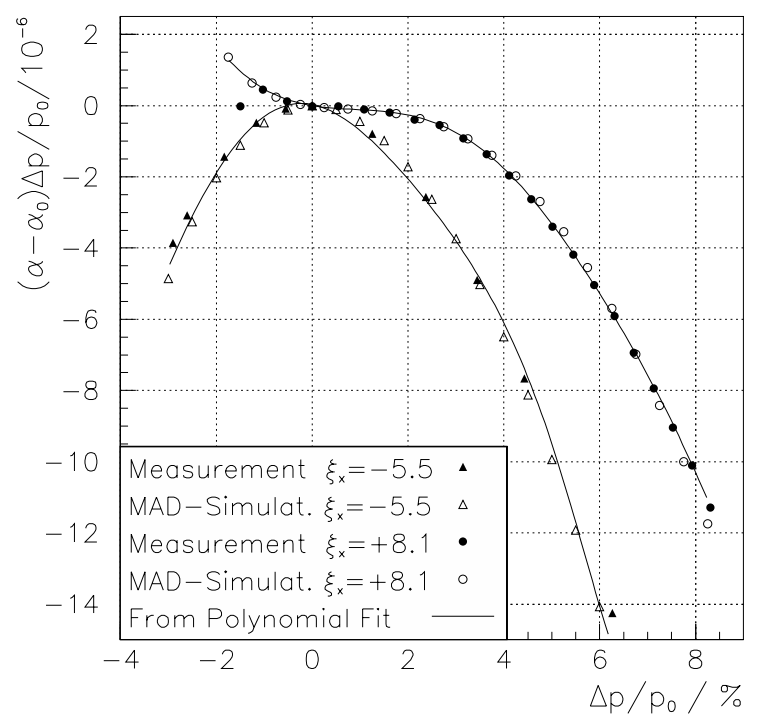

Figure 2: $\left(\alpha-\alpha_{0}\right) \Delta p / p_{0}$ as as a function of the momentum deviation $\Delta p / p_{0}$. Two typical results for $\xi_{x}=+8.1$ and $\xi_{x}=-5.5$ are shown for measured, fitted and simulated data. For the absolute scaling of the horizontal axis $\alpha_{0}=$ $7.34 \cdot 10^{-4}$ was assumed.

Commonly, the coefficients of this last expansion (or a similar one) are abbreviated by $\alpha_{i}$. The first term of this series is by definition $\alpha_{0} \frac{\Delta p}{p_{0}}$. $\alpha_{0}$ can not be extracted from these results, the value $\alpha_{0}=7.3 \cdot 10^{-4}$ was used. For $\alpha$ follows the series expansion:

$$
\frac{\Delta L}{L_{0}} / \frac{\Delta p}{p_{0}}=\alpha=\alpha_{0}+\alpha_{1}\left(\frac{\Delta p}{p_{0}}\right)+\alpha_{2}\left(\frac{\Delta p}{p_{0}}\right)^{2}+\ldots
$$

Fig.3 shows $\alpha / \alpha_{0}$ as a function of the momentum deviation. For a given momentum value this ratio gives the required correction of the rf-frequency, which is of the order of $\pm 30 \%$, due to the influence of higher order terms of the momentum compaction factor.

\section{THE MEASUREMENTS}

The transverse and longitudinal tunes were measured at beam currents of around $10 \mathrm{~mA}$. The coherent beam signal in all 3 directions was measured using a strip line signal and analyzed with a 'Rohde \& Schwarz FSEA' spectrum analyzer. The accuracy of the transverse and longitudinal tune measurements are determined by the line width of the beam tune signal, which was in the order of $0.5 \%$. The vertical chromaticity was not changed and kept at $\xi_{y}=1.6$, only data for different horizontal $\xi_{x}$-values were recorded. The rf-frequency was detuned until beam loss. Beam loss by critical transverse resonances was avoided by readjusting the quadrupole settings if required; it was verified, that the longitudinal tune was not influenced by this. For fixed horizontal chromaticity but different values of vertical chromaticity it was confirmed, that the longitudinal tune is not dependent on the vertical chromaticity. Different cavity voltages showed also no influence on the normalized longitudinal tunes.

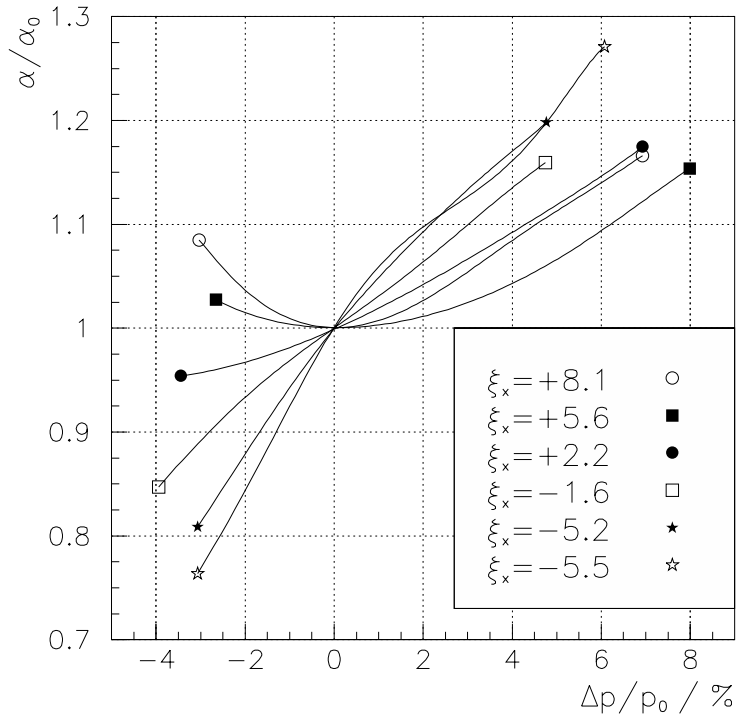

Figure 3: Momentum compaction factor $\alpha / \alpha_{0}$ as a function of the momentum deviation $\Delta p / p_{0}$. Start and end point of each line indicate the range, were stable beam storage is possible. The results of this figure are derived from the polynomial fit.

\section{SUMMARY AND CONCLUSION}

The momentum compaction factor can be measured to a very high accuracy by applying frequency based methods. If fitted by a polynomial, the results shown in Fig.2. would require a polynomial of 5 th order. The value of $\alpha_{0}$ was yet not measured, but taken from a simulation code. In case the momentum acceptance is calculated only with this linear term, the estimated rf-frequency change needs a further correction of the order of $\pm 30 \%$, depending on the chromaticity. As expected, the momentum compaction factor is very sensitive to the horizontal chromaticity. The measured results could be excellent reproduced with the simulation code MAD. From these measurements the momentum limits for stable beam storage was found to be $-4 \%<\Delta p / p_{0}<+8 \%$.

\section{ACKNOWLEDGMENTS}

It is a pleasure to thank Georg Hoffstätter from DESY and Nghiem Phi from Soleil for valuable discussion on this subject.

\section{REFERENCES}

[1] R. Bakker et al., Status and Commissioning-Results of BESSY II, see these proceedings.

[2] Helmut Wiedemann, 'Particle Accelerator Physics', part I and II, Springer-Verlag, Berlin, Germany 1993 and 1995.

[3] H. Grote, F. Ch. Iselin, 'The MAD Program', User's Reference Manual, CERN/SL/90-13 (AP), Geneva, Switzerland, 1994. 